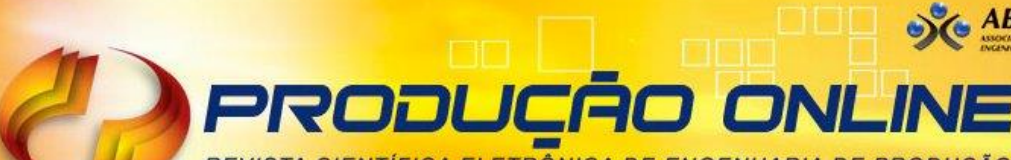 REVISTA CIENTIFICA ELETRÔNICA DE ENGENHARIA DE PRODUÇÃO \\ ISSN 1676-1901
}

\section{BALANCEAMENTO DE OPERAÇÕES E SIMULAÇÃO A EVENTOS DISCRETOS: REDUÇÃO DA OCIOSIDADE DOS OPERADORES EM UMA LINHA DE MONTAGEM}

\section{OPERATIONS BALANCING AND DISCRETE EVENT SIMULATION: REDUCING OPERATOR IDLENESS ON AN ASSEMBLY LINE}

\author{
Flávio Fraga Vilela*E-mail: ffvconsultor@gmail.com \\ Diego Dobscha da Cruz Piedade**E-mail: diegodcpiedade@gmail.com \\ José Arnaldo Barra Montevechi E-mail: montevechi@unifei.edu.br \\ Fabiano Leal* E-mail: fleal@unifei.edu.br \\ *Universidade Federal de Itajubá (UNIFEI), Itajubá, MG \\ **Universidade Federal de Ouro Preto (UFOP), Ouro Preto, MG
}

\begin{abstract}
Resumo: A conduta direcionada a intervenções focadas na redução dos desperdícios nas operações da manufatura e serviços são ações precedentes e imperativas que todo gestor de processos deve buscar constantemente. A diminuição das perdas e desperdícios com consequente aumento da eficiência produtiva se configuram como um dos grandes objetivos das organizações e seus stakeholders. Atualmente, várias técnicas e ferramentas para a redução de desperdícios podem ser empregadas, porém, neste trabalho, será utilizado o conceito do nivelamento do fluxo produtivo, que pode ser alcançado por meio do balanceamento de operações. Portanto, o objetivo do presente trabalho é aplicar a simulação computacional como complemento ao método do balanceamento de operações, reduzindo o número de operadores da linha de montagem e suas ociosidades em um processo fabril real. Como método de pesquisa empregou-se o estudo de caso com a utilização de informações e dados reais, coletadas por meio de uma investigação, de oito meses, realizada na empresa. Por conseguinte, pode-se afirmar que o projeto de balanceamento de operações aplicado na linha de montagem foi eficaz, porquanto uma redução da ociosidade geral para um valor próximo a $4 \%$ foi constatada, conforme relatório da simulação computacional.
\end{abstract}

Palavras-chaves: Balanceamento de Operações. Simulação Computacional. Eficiência Produtiva.

\begin{abstract}
The conduct directed towards interventions focused on reducing waste in manufacturing operations and services are precedent and imperative actions that every process manager must constantly seek. The reduction of losses and waste with consequent increase of productive efficiency is configured as one of the great objectives of organizations and stakeholders. Currently, various techniques and tools for waste reduction can be employed, however, in this work, the concept of production flow leveling, which can be achieved through balancing operations, will be used. Therefore the objective of the present work is to apply computational simulation as a complement to the operations balancing method, reducing the number of assembly line operators and their idleness in a real manufacturing process. As the research method, a case study was used with the use of information and real data collected through an investigation conducted in the company that lasted 8 months. Therefore, it can be stated that the balancing project of assembly line operations was effective since a reduction in overall idleness to a value close to $4 \%$ was found according to the computational simulation report.
\end{abstract}

Keywords: Balancing Operations. Computational Simulation. Productive Efficiency. 


\section{INTRODUÇÃO}

A redução dos desperdícios nas operações de manufatura e serviços são ações precedentes e imperativas que todo gestor deve buscar na conjuntura atual de customização em massa e alta competitividade. Por conseguinte, a diminuição das perdas e desperdícios e o aumento dos ganhos se configuram como um dos principais objetivos das organizações e seus stakeholders. Várias técnicas de redução de desperdícios podem ser empregadas, porém neste trabalho será utilizado o conceito do nivelamento do fluxo, que pode ser alcançado por meio do balanceamento de operações. Segundo Farnes e Pereira (2006), o balanceamento de operações, como método de dimensionamento de capacidade de produção, permite obter melhor aproveitamento dos recursos disponíveis, reduzindo assim alguns desperdícios. Pröpster et al. (2015) afirmam que para que a empresa permaneça competitiva e alcance uma alta produtividade, seu modelo de balanceamento de linhas de montagem precisa lidar com as flutuações e diferentes cenários das variáveis envolvidas. Neste contexto, realizar projetos mediante as incertezas pode ser algo complexo de ser operacionalizado. Para Boysen et al. (2007) e Hu et al. (2011), um dos principais desafios do planejamento da configuração das linhas de montagem é a atribuição de tarefas de montagem às estações, considerando-se todas as restrições dadas pelo produto, linha de montagem e fatores humanos.

Levando-se em consideração o cenário supracitado, o objetivo do presente trabalho é aplicar a simulação computacional como complemento ao método do balanceamento de operações reduzindo o número de operadores da linha de montagem e suas ociosidades. Como método de pesquisa empregou-se o estudo de caso com a utilização de dados reais coletados no decurso de 8 meses.

A seguir a presente pesquisa está organizada em: Revisão Teórica, em que conceitos importantes sobre balanceamento de operações e simulação são apresentados; em Métodos de Pesquisa, a metodologia emprega é detalhada; em Aplicação, são explicitadas de forma sistemática todas as ferramentas aplicadas; por fim, em Resultados, são apresentadas as descobertas mais importantes seguido da Conclusão que finaliza com considerações interessantes sobre a pesquisa. 


\section{REVISÃO TEÓRICA}

A manufatura enxuta (ou Lean) é amplamente adotada desde que a indústria japonesa, liderada pela Toyota, alcançou o topo da indústria automotiva (WOMACK et al., 1992). Segundo Kumar Chakrabortty \& Kumar Paul (2011), lean é um termo para descrever um sistema que produz o que o cliente deseja, quando o deseja, com o mínimo de desperdício, baseado no sistema de produção da Toyota. O pensamento enxuto se concentra no lean de valor agregado e consiste nas melhores práticas, ferramentas e técnicas de todo o setor, com o objetivo de reduzir o desperdício e maximizar o fluxo e a eficiência do sistema geral para alcançar a satisfação final do cliente. A fabricação enxuta é uma filosofia de fabricação que reduz o tempo entre o pedido do cliente e a criação/remessa do produto, eliminando as fontes de desperdício. Para Nahmias (2001), outra maneira de explicar o lean é que ele visa alcançar a mesma saída com menos tempo, menos espaço, menos esforço humano, menos máquinas, menos material e menos custos.

A reconstrução dos fabricantes japoneses após a Segunda Guerra Mundial enfrentava o declínio de recursos humanos, materiais e financeiros. Os problemas que eles possuíam na fabricação eram muito diferentes dos de seus colegas ocidentais. Conforme afirma Monden (1998), essas circunstâncias levaram ao desenvolvimento de novas práticas de fabricação e de menor custo. Então se desenvolveu um sistema de produção disciplinado e focado em processos, agora conhecido como "Sistema Toyota de Produção", "Produção Lean" ou "Manufatura Enxuta". O desperdício é algo que não contribui para transformar uma peça de acordo com as necessidades do cliente. $O$ objetivo da manufatura enxuta é a eliminação de desperdícios em todas as áreas da produção, incluindo relacionamento com clientes, design de produtos, redes de fornecedores e gerenciamento de fábricas.

Ainda hoje, todo tipo de empresa vê a filosofia de manufatura enxuta como uma maneira de permanecer competitiva no mercado (LINDO-SALADOECHEVERRÍA et al., 2015). A manufatura enxuta concentra-se na eliminação absoluta dos desperdícios (OHNO, 1997). Para eliminar todos os tipos de desperdício e suas causas, um conjunto de ferramentas e conceitos foi desenvolvido, como o 5S, sistemas pull/push Kanbans, tempo takt, manutenção produtiva total (TPM), troca rápida de setup e balanceamento de operações. 
Um sistema de produção eficaz requer um ambiente de trabalho organizado, limpo e sustentável. O 5 S é uma ferramenta organizacional para classificar, estabilizar, limpar, padronizar e sustentar ambientes de trabalho (OSADA, 1992). Em seguida, também é importante entender a diferença entre os sistemas push e pull. Nos sistemas push, a produção ocorre de forma independente e individual em cada processo, e não há comunicação entre esses processos em relação às suas demandas. Essa falta de comunicação pode resultar em superprodução (OHNO, 1997). Nos sistemas pull, por outro lado, a comunicação é estabelecida ao longo do fluxo do processo, para que a produção ocorra "just-in-time" (ROTHER \& SHOOK, 1999).

A comunicação do processo pode ser estabelecida com o uso de Kanbans. Kanban é uma informação (um cartão, um espaço no chão, uma mensagem eletrônica) automaticamente enviada e usada no controle de produção (LAGE JUNIOR \& GODINHO FILHO, 2008). O Kanban envia informações sobre a demanda (tipo e quantidade de peças) e onde e quando deve ser entregue. De fato, a demanda desempenha um papel fundamental nos sistemas lean. A produção e o ritmo de produção devem ser acionados pelas demandas dos clientes finais. Por esse motivo, a taxa de saída de cada processo deve permanecer o mais próximo possível do tempo takt, que é o tempo de produção disponível dividido pela demanda no mesmo período (ROTHER \& SHOOK, 1999).

O tempo real de produção disponível geralmente é comprometido por dois problemas: falha no processo e execução da instalação. Para superar o primeiro problema, é aplicada a manutenção produtiva total. O TPM é o envolvimento geral da equipe no processo de manutenção produtiva, que envolve identificação e estudo de falhas, capacitação e melhoria contínua (NAKAJIMA, 1989). A segunda questão está relacionada à flexibilidade do sistema. Hoje, há uma demanda crescente por produtos personalizados. Além disso, muitas estações de trabalho são projetadas para produzir mais de um produto. Portanto, as configurações do processo devem ser executadas o mais rápido possível. A troca rápida de setup é uma teoria e um conjunto de técnicas usadas para obter reduções neste tempo de setup (SHINGO, 1985). O balanceamento de operações tem como objetivo equalizar o fluxo de produção reduzindo os desperdícios inerentes ao processo.

Sobre o balanceamento da linha de montagem, pode-se dizer que foi proposto pela primeira vez em 1961 (HELGESON, 1961). De acordo com Sime, 
Jana \& Panghal (2019), uma linha de montagem é um processo de fabricação no qual as peças são adicionadas à medida que o produto semiacabado se move de uma estação de trabalho para a próxima, onde as peças são adicionadas em sequência até que o produto final seja obtido. Segundo Hicks (2007), um dos principais objetivos da análise da linha de montagem em indústrias é minimizar tanto a escassez de produtos como o estoque excessivo.

Segundo Rauf et al. (2020), no Problema de Balanceamento da Linha de Montagem (Assembly Line Balancing Problem - ALBP), várias tarefas e operações são atribuídas a diferentes estações, otimizando o tempo do ciclo, equilibrando a eficiência e o índice de suavidade etc.

Uma definição essencial e primária dentro do contexto do balanceamento de operações é o tempo de ciclo, que é predeterminado por uma taxa de produção desejada, de maneira que a quantidade desejada de produto final seja produzida dentro de um determinado período de tempo (GRZECHCA, 2011). Para Zupan \& Herakovic (2015), nesse sentido, um dos principais problemas é como organizar as tarefas na linha de produção a serem executadas. Uma maneira eficaz de atingir esse objetivo é equilibrar as linhas de montagem e as células de trabalho. Dessa forma, o balanceamento de operações é um importante método de eficácia comprovada que pode ajudar a melhorar o rendimento das linhas de montagem e células de trabalho, reduzindo os custos e os requisitos de mão-de-obra. Logo, o balanceamento de linha de produção significa o nivelamento ou a atribuição de operações de trabalho às estações de trabalho e à carga de trabalho em todas as operações ao longo do processo produtivo, de forma que a atribuição seja ótima e remova o gargalo.

De acordo com Sime et al. (2019), para que se execute com eficiência o processo de balanceamento de linha, é necessária uma boa observação do sistema geral. No entanto, a rapidez com que todo o processo ocorre, o grande número de variáveis do sistema e a natureza estocástica dessas variáveis tornam muito difícil para um ser humano observar os sistemas de fabricação reais. Além disso, também é muito difícil resolver manualmente problemas de balanceamento de linha levando em consideração todas as variáveis que afetam o desempenho dos sistemas.

Vários pesquisadores desenvolveram algoritmos específicos para a simplificação e a otimização de problemas de balanceamento de linhas (KHANNA; JANA, 1997; HEGAZY, 1999; SHARMA et al., 2004; LAPIERRE et al., 2006). 
Conforme Schwab (2017), os métodos de simulação são utilizados há várias décadas, sendo usados para descrever, pesquisar e projetar sistemas econômicos e sociais. Atualmente, elas são uma das principais tecnologias dentro da estrutura do conceito da indústria 4.0. Pawlewski (2018) afirma que esse conceito foi criado na Alemanha e define uma nova organização de fábricas (chamadas fábricas inteligentes), permitindo melhor atendimento ao cliente por meio de uma enorme flexibilidade e otimização de recursos.

Segundo Banks et al. (2005), inúmeras são as vantagens proporcionadas pela simulação, embora esta também apresente algumas desvantagens. Pinho (2008) e Torga (2007) afirmam que, se comparada a modelos matemáticos, as vantagens proporcionadas pela simulação a eventos discretos são enormes. Principalmente, no que diz respeito à sua conceituação de fácil compreensão e à possibilidade de comparação virtual, trazendo maiores contribuições para o objeto em estudo.

Segundo Law (2009), a simulação é uma alternativa à experimentação direta no sistema real, evitando, assim, os custos devidos à experimentação real e à interrupção do fluxo de atividades do sistema real. Chwif e Medina (2010) ressaltam que, devido à grande complexidade dos modelos de manufatura, o que ocorre devido à sua natureza dinâmica e aleatória, um modelo de simulação permite reproduzir em um computador o mesmo comportamento que o sistema teria se submetido às mesmas condições de contorno.

Segundo Reeb e Leavengood (2003), a simulação deve ser utilizada quando não é possível realizar uma experimentação com o sistema real, quando outros modelos não funcionariam, quando é necessário analisar como os sistemas operam em um determinado período de tempo ou deseja-se comparar projetos alternativos.

De acordo com Greasley (2003), a animação da simulação pode ser utilizada para comunicar para os diretores, clientes e funcionários o "antes" e o "depois" da implantação de um novo projeto, mostrando como as mudanças afetarão o processo na prática. Os mesmos autores afirmam também que, a animação da simulação pode ser utilizada para treinar funcionários diante de uma nova operação do sistema e permitir que eles criem uma visão geral dos benefícios da mudança, a partir da visão do modelo em execução.

Além de todas as vantagens citadas, Banks et al. (2005) destacam: 
- Novos projetos de equipamentos, definições de layouts e sistemas de transportes podem ser testados via simulação, sem consumo de recursos ou aquisição de equipamentos;

- Fornecer uma maior compreensão sobre interações entre variáveis e a importância de seus efeitos no sistema;

- Análises de gargalo, podem ser realizadas para se descobrir onde estoque em processo, informações e materiais estão em excesso;

- O estudo de simulação pode auxiliar no entendimento de como o sistema opera como um todo, ao invés de apenas como operam suas partes;

- Questões "What if" podem ser respondidas, o que é particularmente útil no design de novos sistemas.

Segundo Carson (2004), frequentemente a simulação consome tempo, os dados não estão disponíveis ou são caros de se obter, e o tempo disponível para as tomadas de decisões não são suficientes para um estudo seguro. Em algumas situações, as animações e outras exibições visuais, combinadas com a pressão de tempo em todos os projetos, podem enganar os tomadores de decisão com conclusões prematuras baseado em evidências insuficientes.

Além disso, vale ressaltar que, a simulação, por mais que apresente algumas restrições de aplicação, se usada corretamente é uma força poderosa para a aprendizagem organizacional, segundo Stansfield et al. (2014). Embora, os vendedores de softwares de simulação tenham desenvolvido pacotes que exigem somente a entrada de dados para sua operação (modelos genéricos), simulação não é só conhecimento de software. Um estudo de simulação é uma atividade sofisticada de análise do sistema que exige que o analista tenha pelo menos um mínimo de conhecimento do método de simulação (validação de modelos, seleção de distribuições de probabilidade para entrada de dados, projeto e análise de experimentos simulados etc.), teoria da probabilidade, estatística, gerenciamento de projetos, e conhecimento detalhado das operações do sistema a ser estudado (LAW, 2003). Assunpção e Jacobs (2019) afirmam que a simulação é uma ferramenta versátil com a possibilidade de aplicações variadas no campo produtivo.

Para Sime, Jana \& Panghal (2019), técnicas de simulação a eventos discretos também foram empregadas em muitas organizações de manufatura para projetar, desenvolver, implementar e analisar o problema de interesse do sistema de 
manufatura. Pedgen et al. define simulação como "o processo de projetar um modelo de um sistema real e realizar experimentos com esse modelo com o objetivo de entender o comportamento do sistema e avaliar várias estratégias para o sistema operacional". Recentemente no Brasil, por exemplo, De Carvalho et al. (2016), utilizaram Otimização via Simulação a Eventos Discretos em casos da área médica com técnicas de economia de esforço computacional, e Assunpção e Jacobs (2019), por sua vez, utilizaram-se da Simulação a Eventos Discretos sob a ótica da Teoria das Restrições para avaliar layouts em uma indústria de alimentos, e ambos obtiveram resultados bastante satisfatórios.

Segundo Pröpster et al. (2015), o registro completo de todos os fatores influentes e suas inter-relações é o pré-requisito para obter resultados de simulação relevantes para decisões por um lado e para fazer alterações e analisar seus efeitos por outro. O uso da simulação permite um prognóstico amplamente correto da utilização da equipe. Ao mapear as inter-relações dependentes do tempo, já é possível avaliar gargalos e avaliar a flexibilidade da equipe antes da realização. Um pré-requisito é a adoção contínua dos dados atuais do plano e da sequência que podem ser usados pelo planejador para analisar os efeitos das sequências de pedidos pendentes, bem como definir e simular configurações do próprio sistema de produção e cenários de carregamento de pedidos.

\section{MÉTODO DE PESQUISA}

O trabalho é de natureza aplicada, pois possui objetivos específicos por meio do desenvolvimento de novos ciclos produtivos ou orientação produtiva para as necessidades e demandas das operações de manufatura e serviço. Para atingir o resultado esperado no contexto do objeto de estudo, a pesquisa possui um objetivo explicativo, uma vez que aprofunda o conhecimento da realidade e explica o porquê das coisas (TURRIONI; MELLO, 2012) mediante uma abordagem qualitativa baseada em um estudo de caso, conforme indica a Figura 1. 
Figura 1 - Metodologia de pesquisa em Engenharia de Produção

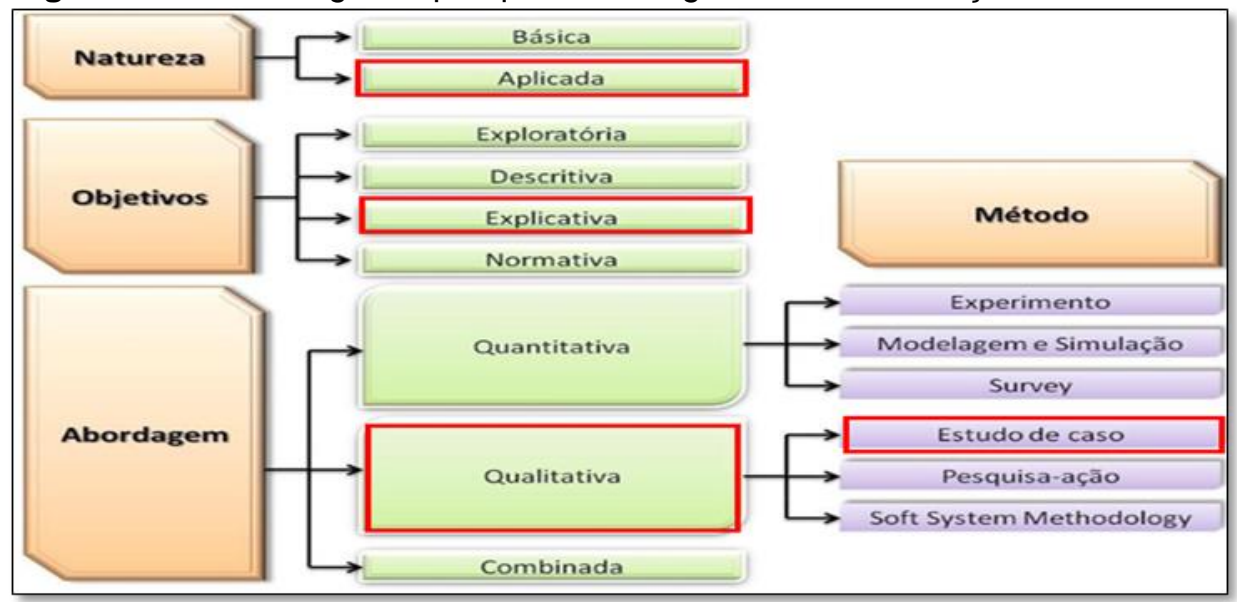

Fonte: Adaptado de Turrioni e Mello (2012)

Nesse estudo de caso, dados reais, informações e fluxos processuais foram coletados da empresa alvo dos estudos. Alguns dados importantes como o tempo de processamento das atividades estudadas foram obtidos por meio de uma sistemática associada à cronoanálise. É essencial frisar, também, que os resultados e outputs dos modelos de simulação computacional foram todos validados por líderes e especialistas da linha de produção. Dessa forma, pode-se atribuir uma alta confiabilidade das conclusões expostas no final da presente pesquisa.

\section{APLICAÇÃO}

\subsection{Objeto de estudo}

A empresa estudada fornece seus produtos para as principais montadoras de automóveis do país e trabalha com um alto volume produtivo o ano todo. $\mathrm{Na}$ condução da presente pesquisa, durante várias semanas foi feito um trabalho de investigação e levantamento de dados, para que o objetivo proposto fosse alcançado. Os dados coletados incluem principalmente os tempos de processamento das operações obtidos por meio da cronoanálise e a lógica de processamento apresentada pelo método de mapeamento de processos IDEF-SIM (LEAL, 2003). Sobre a medição dos tempos de processamento, pode-se dizer que a sistemática empregada da cronoanálise é uma forma de mensurar o trabalho por meio de métodos estatísticos, permitindo calcular o tempo padrão que é utilizado para determinar a capacidade produtiva da empresa, elaborar programas de 
produção e projetos de balanceamento de operações (PEINADO; GRAEML, 2007). As outras informações necessárias para a construção do projeto de balanceamento de operações e simulação computacional foram: número de operadores na linha de montagem, tempo disponível, paradas necessárias como almoço e ginástica laboral, tempo de manutenção preventiva e corretiva por máquina, demanda diária da produção (takt-time) e fluxo das informações no processo.

\subsection{Mapeamento do processo}

Conforme supracitado, a técnica de mapeamento utilizada é conhecida como IDEF-SIM (LEAL, 2003). A Figura 2 a seguir mostra essa técnica aplicada ao contexto estudado:

Figura 2 - IDEF-SIM do processo geral de manufatura

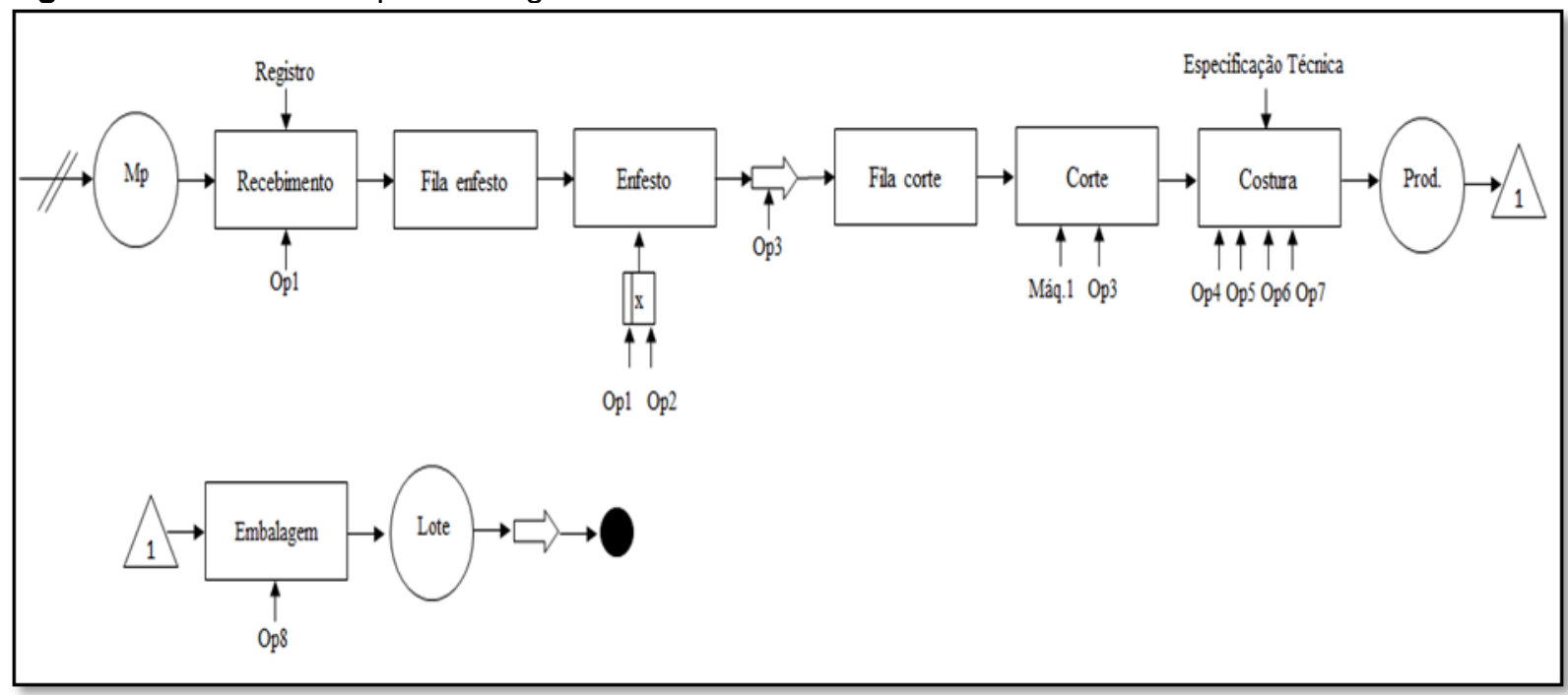

Fonte: Os autores

O fluxo indica que a matéria-prima ( $\mathrm{Mp}$ ) chega ao recebimento sendo acomodada e registrada pelo operador de processo Op1. Em seguida a Mp vai para a fila de enfesto. Após uma espera nessa fila, a Mp segue para a etapa de enfesto. Nesta etapa o tecido será esticado para verificação de algum possível defeito do fabricante, possibilitando identificar anormalidades que não atendam aos padrões estabelecidos pela qualidade. Para executar a referida atividade, dois operadores (Op1 ou Op2) estão aptos, dependendo de suas respectivas disponibilidades. Logo após, a Mp é transportada para fila de corte, por meio do Op3, onde aguarda até ser processada pela etapa de corte. Neste último processo, uma máquina (Máq.1) sendo 
auxiliada por um operador (Op3) desenvolve o processo de leitura dos markers para identificar as peças que serão cortadas. Após esta etapa tem-se a entidade produto (Prod.) concebida que segue para o próximo posto de trabalho que é a costura. Neste local, os tecidos são costurados conforme especificação técnica de modelo e tipo, gerando por fim a capa automotiva. O projeto de balanceamento é desenvolvido nesta etapa. Finalmente o produto Capa Automotiva segue para embalagem, dando fim ao processo mapeado.

\subsection{Situação atual da linha de produção}

$\mathrm{Na}$ situação anterior ao balanceamento da linha de produção têm-se as seguintes métricas, sendo o processo organizado conforme Tabela 1.

\begin{tabular}{ccccc}
\multicolumn{6}{c}{ Tabela 1 - Situacional antes do balanceamento } \\
\hline Métricas & $\begin{array}{c}\text { Posto } \\
\text { Trabalho I }\end{array}$ & $\begin{array}{c}\text { Posto } \\
\text { Trabalho II }\end{array}$ & $\begin{array}{c}\text { Posto } \\
\text { Trabalho III }\end{array}$ & $\begin{array}{c}\text { Posto } \\
\text { Trabalho IV }\end{array}$ \\
\hline & 1 & 12 & 8 & 29 \\
& 2 & 13 & 9 & 24 \\
Noop. & 3 & 14 & 10 & 25 \\
& 4 & 15 & 17 & 26 \\
& 5 & 16 & 18 & 27 \\
& 6 & & 19 & 28 \\
n & 7 & & 20 & 23 \\
TL & 11 & & 21 & 22 \\
TC & 8 & 5 & 8 & 8 \\
F & 752,7 & 229,2 & 347 & 323,2 \\
Nt & 315,3 & 768 & 768 & 768 \\
\hline
\end{tabular}

Fonte: Os Autores

Ao posto de trabalho I estão alocadas 8 operações de produção $(n=8)$, contemplando um tempo total (TL) de 452,7 segundos, sendo o tempo de ciclo (TC) igual a 768 segundos. O cálculo do TC será evidenciado logo a seguir. Por fim, é válido ressaltar que a folga $(F)$ deste referido posto será de 315,3 e o número de trabalhadores $(\mathrm{Nt})$ responsáveis desta etapa será apenas um. A mesma leitura lógica deve ser empregada aos postos remanescentes.

\subsection{Aplicação do método de balanceamento}

O projeto de balanceamento de operações será aplicado à etapa de costura, 
pois é o gargalo de todo o processo produtivo apresentado na Figura 1. A seguir, a Tabela 2 mostra 29 operações e os respectivos tempos padrões. Nesta tabela o número da operação de costura ( $\mathrm{N}^{\circ} \mathrm{Op}$.) estão listados de 1 a 29 e o tempo padrão (TP) de cada operação está em segundos. O procedimento proposto por Slack et al. (2002) foi aplicado para se efetuar os cálculos desse tempo padrão. Vale frisar que um fator de tolerância de $14 \%$ foi aplicado ao contexto da linha de operação estudada seguindo os critérios definidos por Barnes (2008).

\begin{tabular}{cccccc}
\multicolumn{7}{c}{ Tabela 2 } & operações e tempos da costura \\
\hline №Op. & TP & №p. & TP & №Op. & TP \\
\hline 1 & 52,12 & 11 & 60,19 & 21 & 37,62 \\
2 & 37,4 & 12 & 37,21 & 22 & 39,67 \\
3 & 39,67 & 13 & 37,21 & 23 & 37,62 \\
4 & 38,3 & 14 & 55,54 & 24 & 40,08 \\
5 & 88,81 & 15 & 45,69 & 25 & 38,85 \\
6 & 82,65 & 16 & 53,57 & 26 & 49,11 \\
7 & 53,57 & 17 & 39,67 & 27 & 39,67 \\
8 & 45,96 & 18 & 37,62 & 28 & 37,62 \\
9 & 46,65 & 19 & 47,06 & 29 & 40,56 \\
10 & 52,83 & 20 & 39,67 & & \\
\hline
\end{tabular}

Fonte: Os Autores

A Tabela 3 mostra o tempo total e todas as pausas envolvidas no processo produtivo em questão, seguido das justificativas. Também fica evidenciado na última linha dessa tabela o tempo disponível para produção. Essa informação é necessária para se efetuar o cálculo do tempo de ciclo (TC) por etapa, que neste caso é o próprio takt-time (TT), pois a demanda se refere a uma venda consolidada.

Tabela 3 - Tempo disponível de produção

\begin{tabular}{ll}
\hline Tempo total & 616 minutos \\
\hline Ginástica laboral & 22 minutos \\
Reunião & 20 minutos \\
Manutenção preventiva & 15 minutos \\
Refeição & 60 minutos \\
Tempo disponível para produção & 499 minutos \\
\hline
\end{tabular}

Fonte: Os Autores

Considerando uma demanda do turno de 39 kits para a capa automotiva e de acordo com Slack et al. (2002) o tempo de ciclo será de aproximadamente 768 segundos/kit, conforme resultado da Equação 1.

$$
\mathrm{TC}=\frac{\text { tempo disponível para produção do turno } t}{\text { demanda do turno } \mathrm{t}}
$$


Após se calcular o tempo de ciclo (TC), o próximo passo é determinar o número mínimo ( $\mathrm{N}$ mín) de postos de trabalho para a realização do balanceamento de operações, conforme a Equação 2 (REGINATO et al., 2016; SLACK et al., 2002).

$$
\mathrm{N}_{\text {min }}=\frac{\sum \text { tempos individuais de cada tarefa }}{\mathrm{TC}}
$$

Logo, pela Equação 2, chega-se no valor de $\mathrm{N}_{\min }=2$ postos de trabalho. $\mathrm{A}$ Tabela 4 aponta o tipo de precedência (Prec.) por operação (№Op.).

\begin{tabular}{cccccc}
\multicolumn{6}{l}{ Tabela $4-$ precedência linear das operações } \\
\hline №Op. & Prec. & NoOp. & Prec. & №Op. & Prec. \\
\hline $\mathbf{1}$ & - & $\mathbf{1 1}$ & - & $\mathbf{2 1}$ & 20 \\
$\mathbf{2}$ & 1 & $\mathbf{1 2}$ & - & $\mathbf{2 2}$ & 21 \\
$\mathbf{3}$ & 2 & $\mathbf{1 3}$ & - & $\mathbf{2 3}$ & - \\
$\mathbf{4}$ & 3 & $\mathbf{1 4}$ & - & $\mathbf{2 4}$ & 23 \\
$\mathbf{5}$ & 4 & $\mathbf{1 5}$ & - & $\mathbf{2 5}$ & 24,20 \\
$\mathbf{6}$ & 5,2 & $\mathbf{1 6}$ & - & $\mathbf{2 6}$ & 25 \\
$\mathbf{7}$ & 6 & $\mathbf{1 7}$ & 16 & $\mathbf{2 7}$ & 26 \\
$\mathbf{8}$ & 7 & $\mathbf{1 8}$ & 17 & $\mathbf{2 8}$ & 27 \\
$\mathbf{9}$ & 8 & $\mathbf{1 9}$ & 18,11 & $\mathbf{2 9}$ & - \\
$\mathbf{1 0}$ & $\mathbf{9}$ & $\mathbf{2 0}$ & 19 & & \\
\hline
\end{tabular}

Fonte: Os Autores

Como procedimento do balanceamento de operações (GERHARDT et al., 2007), na Tabela 5, agrupou-se as 29 operações em 2 postos de trabalho de forma que as precedências apontadas na Tabela 4 fossem todas respeitadas juntamente com o tempo de ciclo (768 segundos). Quando ocorre de existir mais de uma possibilidade de balanceamento de operações que respeite as premissas acima, deve-se escolher aquela que retorna o menor tempo de folga $(F)$ por posto de trabalho (PT).

Tabela 5 - balanceamento de operações

\begin{tabular}{cccc}
\hline \multicolumn{2}{c}{$\begin{array}{c}\text { Posto Trabalho I } \\
\text { Noop. }\end{array}$} & \multicolumn{2}{c}{$\begin{array}{c}\text { Posto Trabalho II } \\
\text { Nºp. }\end{array}$} \\
\hline 1 & 12 & 8 & 22 \\
2 & 13 & 9 & 29 \\
3 & 14 & 10 & 24 \\
4 & 15 & 17 & 25 \\
5 & 16 & 18 & 26 \\
6 & & 19 & 27 \\
7 & & 20 & 28 \\
11 & & 21 & 23 \\
$\mathbf{n}$ & $\mathbf{1 3}$ & $\mathbf{n}$ & $\mathbf{1 6}$ \\
TL & $\mathbf{6 8 1 , 9}$ & TL & $\mathbf{6 7 0 , 2}$ \\
TC & $\mathbf{7 6 8}$ & TC & $\mathbf{7 6 8}$ \\
F & $\mathbf{8 6 , 1}$ & F & $\mathbf{9 7 , 8}$ \\
\hline
\end{tabular}

Fonte: Os Autores 
No posto de trabalho I foram alocadas 13 operações $(n=13)$ perfazendo um tempo total (TL) de 681,9 segundos. Como o tempo de ciclo é 768 segundos, obtemos uma folga $(F)$ de 86,1 para este posto de trabalho.

\subsection{Utilização da Simulação a Eventos discretos}

A aplicação da simulação terá o objetivo de validar os resultados obtidos por meio do método de balanceamento supracitado. Também irá apontar por meio de seus relatórios o nível dos estoques intermediários e utilização dos recursos envolvidos e atuantes na linha de produção considerada nesta pesquisa. Por conseguinte, a simulação computacional será aplicada na linha de produção antes e depois do balanceamento de operações.

Sobre a confiabilidade dos resultados dos dois modelos computacionais apresentados, pode-se dizer que ambos têm uma alta confiança, pois foram validados por meio do teste de Turing ou validação black-box (CHWIF; MEDINA, 2010; KLEIJNEN, 1995; SARGENT, 2004). Neste teste de validação, escolhe-se uma pessoa que entenda bem do sistema (um especialista ou líder do processo) que foi simulado e apresentam-se a ele dois conjuntos de dados de saída, um vindo do sistema real e outro do modelo de simulação. Geralmente esses dados de saída se referem à quantidade produzida, tamanho do estoque intermediário (work-inprocess) etc. Caso o especialista não consiga distinguir se os resultados são oriundos do sistema real ou do modelo de simulação computacional, então o modelo pode, em certa extensão, ser considerado "válido". Isso porque os dados estão equivalentes e quase iguais e o líder não consegue diferenciá-los.

A Figura 3 exibe o modelo computacional da linha de produção antes do balanceamento feito usando-se o software FlexSim ${ }^{\circledR}$. Para conceber este modelo de simulação foram considerados os dados das Tabelas 1 e 3 . É válido ressaltar que os tempos de processamento dos quatro operadores foram considerados em sua forma determinística. 
Figura 3 - Modelo computacional antes do balanceamento

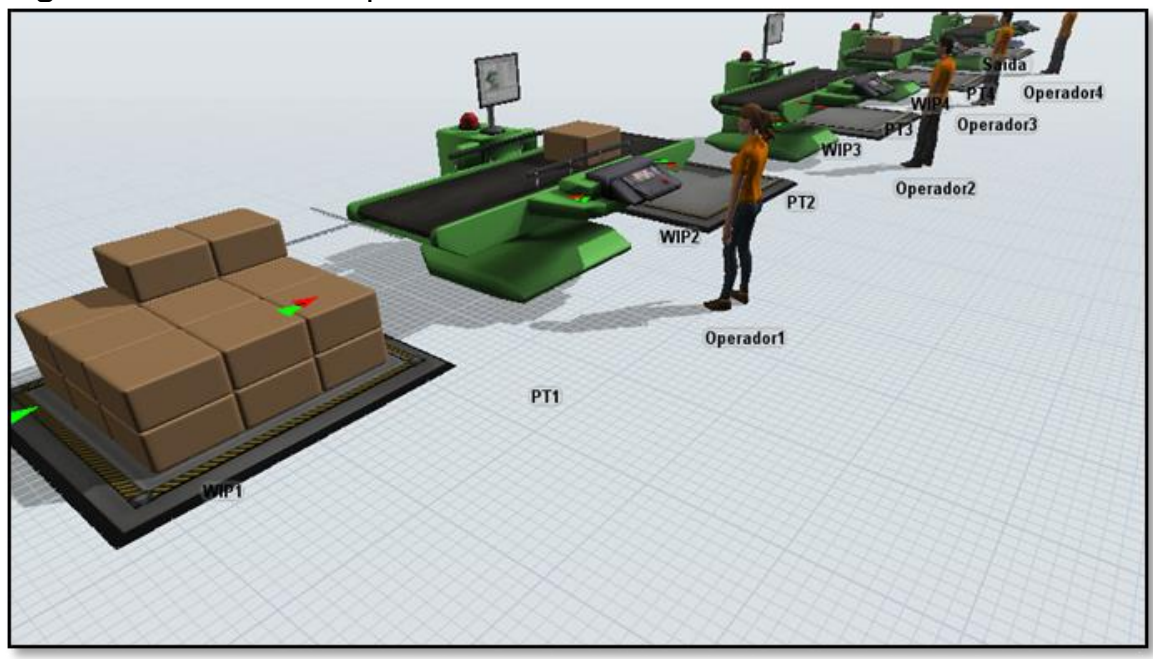

Fonte: Os Autores

Portanto, neste modelo computacional, fica evidenciada a situação já comentada na Tabela 1, onde quatro operadores trabalham na linha de produção e o processo está desbalanceado. É justamente nesta falta de nivelamento do processo que existe uma lacuna para ser explorada, no que tange à redução de algum desperdício como preconiza o sistema Lean Production.

A seguir, na Figura 4, tem-se o modelo computacional da linha de produção após aplicação do balanceamento de operações. Fica bem evidente que temos agora dois postos de trabalho com um operador trabalhando em cada. Sendo que, no posto de trabalho I ficaram alocadas 13 operações e no posto de trabalho II ficaram 16 operações. As informações sobre o detalhamento das operações já foram apresentadas e estão disponíveis na Tabela 5. É essencial destacar que o estoque intermediário entre os dois postos de trabalho é de aproximadamente igual a zero, conforme relatório do simulador computacional. Este fato corrobora uma das principais premissas do balanceamento de operações que é manter o fluxo produtivo entre as etapas nivelado e equalizado. Este valor de "zero" estoque intermediário também indica que o método do balanceamento foi corretamente aplicado. 
Figura 4 - Modelo computacional após o balanceamento

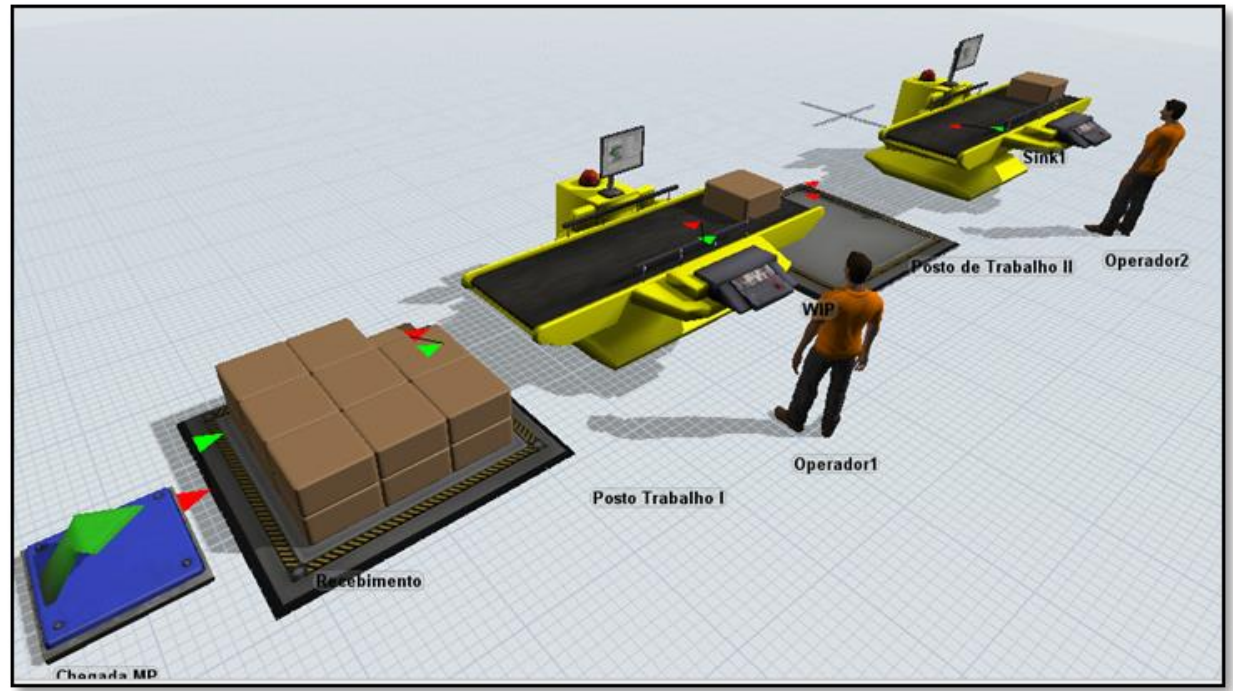

Fonte: Os Autores

\section{RESULTADOS}

É de extrema importância o emprego de algumas métricas finais para que comparações possam ser feitas no que tange à efetividade do projeto de balanceamento de operações. Portanto, será aplicada a Equação 3 para aferir os ganhos. Esta equação mede a eficiência global do balanceamento (ZUPAN; HERAKOVIC, 2015).

$\varepsilon=\frac{\sum \text { tempos individuais de cada tarefa }}{\mathrm{TC} \times \mathrm{Nmín}}$

Os resultados encontrados estão exibidos na Tabela 6, em que também foi inserida a variável folga total $(\mathrm{Ft})$. Logo, é possível constatar que a aplicação do método do balanceamento de operações aumentou o valor da eficiência global em $100 \%$ com consequente redução da folga total em aproximadamente $85 \%$.

Tabela 6 - Comparação dos resultados

\begin{tabular}{ccc}
\hline Var & $\begin{array}{c}\text { Antes do } \\
\text { balanceamento }\end{array}$ & $\begin{array}{c}\text { Depois do } \\
\text { balanceamento }\end{array}$ \\
\hline$\varepsilon$ & $44 \%$ & $88 \%$ \\
Ft & 1279 segundos & 184 segundos \\
\hline
\end{tabular}

Fonte: Os Autores 
Na Figura 5 seguinte, tem-se a informação percentual da utilização (verde) e ociosidade (vermelho) dos quatro operadores antes do balanceamento. Nota-se que o operador 2 é o mais ocioso, apresentando um valor percentual de quase $51 \%$. Continuando a análise, o operador 3 , apresenta um valor de ociosidade próximo à $26 \%$, seguido do operador 4 com quase $31 \%$. Estes valores significativos encontrados por meio do dashboard do FlexSim ${ }^{A}$ indicam o grande desperdício que está ocorrendo na operação e, as vezes, fica implícito aos olhos dos gestores menos experientes e preparados.

Figura 5 - Relatório gráfico dos recursos antes do balanceamento

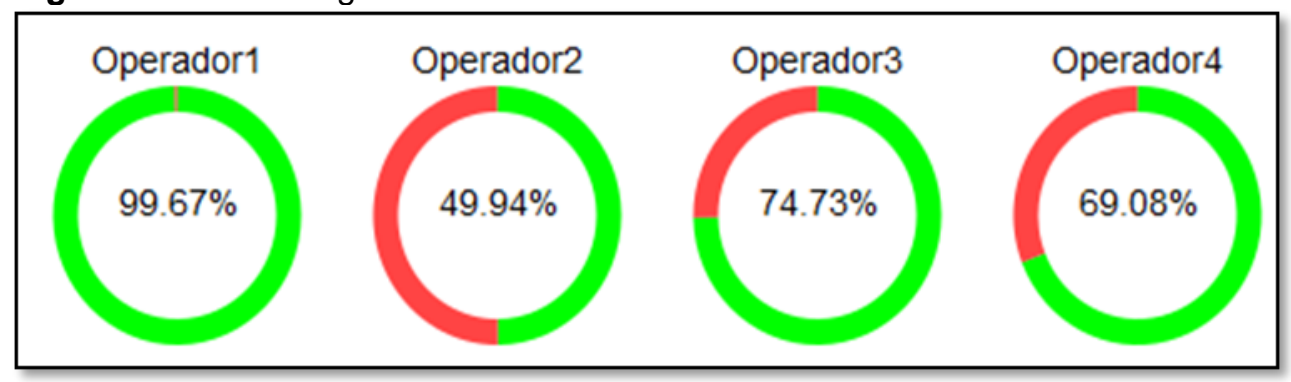

Fonte: Os Autores

Por fim, na Figura 6 abaixo, tem-se novamente as informações sobre utilização e ociosidade dos dois operadores depois do balanceamento de operações. Fica nítida a grande melhoria proveniente da aplicação do método de equalização dos fluxos. Nota-se, por meio do report do software de simulação que a ociosidade do operador 1 é igual "zero" e a ociosidade do operador 2 é de apenas 4\%. Ou seja, a ociosidade total da linha de montagem é de $4 \%$. Isso demonstra que o desperdício ociosidade foi interpelado corretamente conforme premissas do Lean. Desta forma a eficiência do processo fica evidenciada, sendo que investimentos significativos não foram feitos, o que comprova a eficácia da racionalização do processo.

Figura 6 - Relatório gráfico dos recursos após o balanceamento

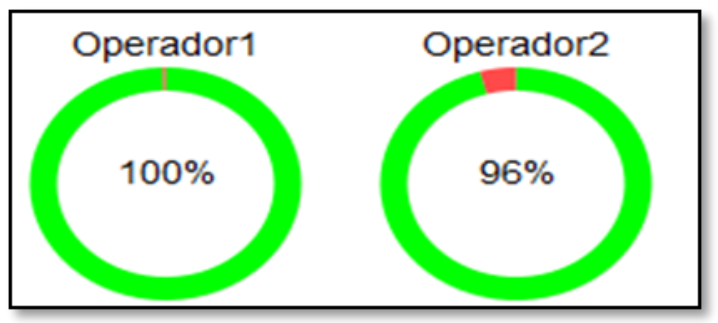

Fonte: Os Autores 
Portanto, nesta última figura, é notório que além da redução de dois operadores na linha de montagem, o nível de ociosidade total dos recursos após o balanceamento ficou em apenas 4\%, corroborando desta forma a efetividade do método empregado.

\section{CONCLUSÕES}

O objetivo proposto no presente trabalho foi alcançado, pois a simulação a eventos discretos foi aplicada como complemento ao projeto de balanceamento da linha de produção e evidenciou resultados relevantes sobre a utilização e ociosidade dos operadores na linha de montagem. Outro resultado relevante alcançado foi a redução de dois operadores na linha de montagem, respeitando-se o tempo de ciclo do processo, seguido da diminuição das suas respectivas ociosidades. Sendo assim, pode-se afirmar que o projeto de balanceamento de operações na linha de costura foi eficaz, porquanto a eficiência global do balanceamento mostrou um aumento de $100 \%$ e o percentual de ociosidade total dos operadores após o balanceamento diminuiu para um valor de $4 \%$.

\section{AGRADECIMENTOS}

Os autores agradecem a CAPES, CNPq e FAPEMIG pelo apoio e suporte.

\section{REFERÊNCIAS}

ASSUNPÇÃO, Lucas Eggers; JACOBS, William. Estudo comparativo entre layouts sob a ótica da teoria das restrições com apoio de simulação de eventos discretos em empresa de alimentos. Revista Produção Online, v. 19, n. 1, p. 152-178, 2019.

https://doi.org/10.14488/1676-1901.v19i1.3147

BANKS, J., J. S. CARSON, B. L. NELSON, D. M. NICOL. Discrete-event Simulation. 4. ed. Upper Saddle River, NJ: Prentice-Hall, 2005.

BARNES, R.M - Estudo de movimentos e tempos: projeto e medida do trabalho. 6. ed. São Paulo: Blucher, 2008.

BOYSEN, N., FLIEDNER, M., SCHOLL, A. A classification of assembly line balancing problems. European Journal of Operational Research, n. 183, p. 674-693, 2007. https://doi.org/10.1016/j.ejor.2006.10.010 
CARSON, J. S. Introduction to modeling and simulation. In: WINTER SIMULATION CONFERENCE. [Anais...]. Washington, D.C., USA, 2004.

https://doi.org/10.1109/WSC.2004.1371297

CHWIF, L.; MEDINA, A.C. Modelagem e simulação de eventos discretos, teoria \& publicações. 3. ed. São Paulo: Ed do autor, 2010.

DE CARVALHO MIRANDA, Rafael et al. Otimização via simulação por metamodelagem: um estudo em casos da área médica. Revista Produção Online, v. 16, n. 3, p. 1058-1078, 2016. https://doi.org/10.14488/1676-1901.v16i3.2573

FARNES, V. C. F., \& PEREIRA, N. A. Balanceamento de linha de montagem com o uso de heurística e simulação : estudo de caso na linha branca. GEPROS: Gestão Da Produção, Operações e Sistemas, (i), p. 1-12, 2006.

GERHARDT, M. P., FOGLIATTO, F. S., \& CORTIMIGLIA, M. N. Metodologia para o balanceamento de linhas de montagem multi-modelo em ambientes de customização em massa. Gestão e Produção, v. 14, n. 2, p. 267-279, 2006. https://doi.org/10.1590/S0104530X2007000200006

GREASLEY, A. Using business-process simulat ion within a business-process reengineering approach. Business Process Management Journal, v. 9, n. 4, p. 408- 420, 2003. https://doi.org/10.1108/14637150310484481

GRZECHCA, W. Final results of assembly line balancing problem, assembly line: theory and practice. [s.I.]: InTech, 2011. https://doi.org/10.5772/38865

HEGAZY, T. Optimization of resource allocation and levelling using genetic algorithms. Journal of Construction Engineering and Management, v. 125, n. 3, p. 167-175, 1999. https://doi.org/10.1061/(ASCE)0733-9364(1999)125:3(167)

HELGESON, W. B., BIRNIE, D. P. Assembly line balancing using the ranked positional weighting technique, Journal of Industrial Engineering, n. 12, p. 394-398, 1961.

HICKS, B.J. Lean information management: understanding and eliminating waste, International Journal Of Information Management, v. 27, n. 4, p. 233-249, 2007. https://doi.org/10.1016/j.ijinfomgt.2006.12.001

HU, S. J., KO, J., WEYAND, L., ELMARAGHY, H.A., LIEN, T.K., KOREN, Y., BLEY, H., CHRYSSOLOURIS, G., NASR, N., SHPITALNI, M. Assembly system design and operations for product variety. CIRP Annals - Manufacturing Technology, v. 60, n. 2, p. 715-733, 2011. https://doi.org/10.1016/i.cirp.2011.05.004

KHANNA, S. AND JANA, P. A computerized management system for line balancing. New Delhi: NIFT, 1997.

KLEIJNEN, J. P. C. Verification and validation of simulations models. European Journal of Operational Research, v. 82, n.1, 1995. https://doi.org/10.1016/0377-2217(94)00016-6

KUMAR CHAKRABORTTY, Ripon; KUMAR PAUL, Sanjoy. Study and implementation of lean manufacturing in a garment manufacturing company: Bangladesh perspective. Journal of Optimization in Industrial Engineering, n. 7, p. 11-22, 2011. 
LAGE JUNIOR, M., \& GODINHO FILHO, M. Adaptations of the kanban system: review, classification, analysis and evaluation. Gestão \& Produção, v. 15, n. 1, p. 173-188, 2008. https://doi.org/10.1590/S0104-530X2008000100015

LAPIERRE, S. D. RUIZ, A. AND SORIANO, P. Balancing assembly lines with tabu search. European Journal of Operational Research, v. 168, n. 3, p. 826-837, 2006.

https://doi.org/10.1016/j.ejor.2004.07.031

LAW, A. M. How to build valid and credible simulation models. In: WINTER SIMULATION CONFERENCE. [Proceedings...]. Austin, TX, USA, 2009.

https://doi.org/10.1109/EMR.2009.5235457

LAW, A. M. How to conduct a successful simulation study. WINTER SIMULATION CONFERENCE. [Proceedings...]. New Orleans, LA, USA, 2003.

LEAL, F. Um diagnóstico do processo de atendimento a clientes em uma agência bancária através de mapeamento do processo e simulação computacional. 2003. 224 f. Dissertação (Mestrado em Engenharia de Produção) - Universidade Federal de Itajubá, Itajubá, MG, 2003.

LINDO-SALADO-ECHEVERRÍA, C., SANZ-ANGULO, P., DE-BENDITO-MARTÍN, J. J., \& GALINDO-MELERO, J. Aprendizaje del lean manufacturing mediante Minecraft: aplicación a la herramienta 5S. Revista Ibérica de Sistemas y Tecnologías de Información, n. 16, p. $60-75,2015$

MONDEN, Y. Toyota production system: an integrated approach to just-in-time. Norcross, Georgia: Engineering \& Management Press, 1998.

NAHMIAS, S. Production and operations analysis. New York: McGraw Hill, 2001.

NAKAJIMA, S. Introdução ao TPM. São Paulo: IMC Internacional Sistemas Educativos, 1989.

OHNO, T. O sistema Toyota de produção: além da produção em larga escala. Porto Alegre: Artes Médicas, 1997.

OSADA, T. Housekeeping, 5S'S: Seiri, Seiton, Seiso, Seiket Su, Shitsuke; cinco pontoschaves para o ambiente da qualidade total. São Paulo: Iman, 1992.

PAWLEWSKI, P. Using PFEP for simulation modeling of production systems. Procedia Manufacturing, v. 17, p. 811-818, 2018. https://doi.org/10.1016/j.promfg.2018.10.132

PEDGEN, C., SADOWSKI, R., \& SHANNON, R. Introduction to simulation using siman. New York: McGraw-Hill, 1995,

PEINADO, Jurandir; GRAEML, Alexandre Reis. Administração da produção: operações industriais e de serviços. Curitiba: UnicenP, 2007.

PRÖPSTER, M. et al. Validation of line balancing by simulation of workforce flexibility. Procedia CIRP, v. 33, p. 93-98, 2015. https://doi.org/10.1016/..procir.2015.06.018

RAUF, M. et al. A smart algorithm for multi-criteria optimization of model sequencing problem in assembly lines. Robotics and Computer-Integrated Manufacturing, v. 61, p. 101844, 2020. https://doi.org/10.1016/j.rcim.2019.101844 
REEB, J.E.; LEAVENGOOD, S. Introduction and overview for simulating a manufacturing system. Ext. Serv. EM 8837-E, Oregon State Univ. p.12, 2003.

REGINATO, G., ANZANELLO, M. J., \& KAHMANN, A. Mixed assembly line balancing method in scenarios with different mix of products. Gestão e Produção, v. 23, n. 2, p. 294307, 2016. https://doi.org/10.1590/0104-530x1874-14

ROTHER, M., \& SHOOK, J. Aprendendo a enxergar: mapeando o fluxo de valor para agregar valor e eliminar o desperdício. São Paulo: Lean Institute Brasil, 1999.

SARGENT, R. G. Verification and validation of simulation models are discussed in this paper. Journal of Simulation, v. 7, n. 1, p. 12-24, 2013. https://doi.org/10.1057/jos.2012.20

SCHWAB, K. The fourth industrial revolution. Crown Business, 2017.

SHARMA, G. YADUVANSHI, S. AND JANA, P. Computerized allocation of operators in an assembly line in the garment industry. New Delhi: NIFT, 2004.

SHINGO, S. A revolution in manufacturing: the SMED system. Portland: Productivity Press, 1985.

SIME, Haile; JANA, Prabir; PANGHAL, Deepak. Feasibility of Using Simulation Technique for Line Balancing In Apparel Industry. Procedia Manufacturing, v. 30, p. 300-307, 2019. https://doi.org/10.1016/j.promfg.2019.02.043

SLACK, N; CHAMBERS, S; JOHNSTON, R. Administração da Produção. 2. ed. São Paulo: Atlas, 2002.

STANSFIELD, T.; MASSEY, R.; JAMISON, D. Simulation Can Improve Reality: Get More from the Future. Industrial Engineer, v. 46, n. 3, p. 38-42, 2014.

TORGA, B.L.M. Modelagem, simulação e otimização em sistemas puxados de manufatura. 2007. 139 f. Dissertação (Mestrado em Engenharia de Produção) Universidade Federal de Itajubá, Itajubá, MG, 2007.

TURRIONE, J. B., MELLO, C. H. P. Metodologia de pesquisa em engenharia de produção: estratégias, métodos e técnicas para condução de pesquisas quantitativas e qualitativas. Itajubá, Minas Gerais, 2012.

WOMACK, J. P., JONES, D. T., \& ROOS, D. A máquina que mudou o mundo. Rio de Janeiro: Campus, 1992.

ZUPAN, H.; HERAKOVIC, N. Production line balancing with discrete event simulation: A case study. IFAC-PapersOnLine, v. 48, n. 3, p. 2305-2311, 2015.

https://doi.org/10.1016/j.ifacol.2015.06.431

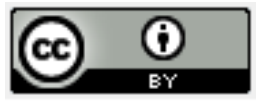

Artigo recebido em: 18/11/2019 e aceito para publicação em: 03/06/2020

DOI: http://dx.doi.org/10.14488/1676-1901.v20i2.3842 